\title{
Factors Associated With Body Weight Status among Students in Infrastructure University Kuala Lumpur (IUKL), Kuala Lumpur, Malaysia
}

Authors

\author{
Salma K. Mahgoub, Hazizi Abu Saad, Rosita Jamaluddin
}

Department of Nutrition and Dietetics, Faculty of Medicine and Health Sciences, Universiti Putra Malaysia, 43400 UPM Serdang, Selangor, Malaysia

\begin{abstract}
The occurrence of obesity and overweight status in the population has caught public attention. This is in line with the World Health Organization's concern (2013). The present study aimed to determine factors associated with body weight status among students in Infrastructure University Kuala Lumpur (IUKL), Kuala Lumpur, Malaysia. A cross-sectional study was conducted to asses physical activity level, environmental factors affecting physical activity level and their association with body weight status among 280 students in Infrastructure University Kuala Lumpur (IUKL), Kuala Lumpur, Malaysia. The mean of respondent's total physical activity per week was $3988.50 \pm 6758.45$ METs minute/week. This was significantly higher $(t=3.020, p=0.003)$ among male respondents $(5496.37 \pm 7860.26$ METs minute/week) compared to females $(3032.93 \pm 5779.55$ METs minutes/week). The overall environmental supports for physical activity among the respondents were at moderate level (2.17 \pm 0.72$)$, although the total environmental supports for physical activity among female is slightly higher compared to male $(2.17 \pm 0.77$ vs $2.15 \pm 0.63)$, no significant different was observed $(t=-0.25 ; p=0.805)$. In general over weight / obese status was highly prevalent among those with low involvement with neighbourhood environment for physical activity (28\%) (the lowest quartile). Although adjusted crude analysis of support to neighbourhood environment for physical activity showed reverse associations with overweight / obese status, no significant trend was observed.
\end{abstract}

\section{Background}

In 2014, 39\% of adults, 18 years and older were overweight (38\% of men and $40 \%$ of women), and $13 \%$ were obese (11\% of men and $15 \%$ of women). This shows that almost 2000 billion adults worldwide are overweight and out of these over 600 million were obese (WHO, 2015).In the WHO Regions of the Americas states the highest rate of obesity and overweight (62\% for overweight in both sexes, and $26 \%$ for obesity) and lowest in the WHO Region for South East Asia (14\% overweight in both sexes and 3\% for obesity).In the WHO region of the Americas and European and Eastern Mediterranean Regions, more than $50 \%$ of women are overweight. And in all these three regions, approximately half of overweight women were obese. $25 \%$ in Europe, 24\% in the Eastern Mediterranean and 30\% in the Americas. Women had nearly double the prevalence of obesity among men in the WHO African, Eastern Mediterranean and South-East Asia Regions (WHO, 2015).

According to Nassreddin, Naja, Chamieh, Adra, Sibai, and Hwallah, (2012), from their study in Lebanon, it shows that the prevalence of overweight was stable from 1997 to 2009 for adults (20 years and above; it was $37 \%$ in 1997 versus $36.8 \%$ in 2009 . However, the same study showed that obesity was increased in 2009 at $28.2 \%$ versus $17.4 \%$ in 1997 . BMI also seems to be higher and increasing in high income countries such as 
USA, with $12 . \mathrm{kg} / \mathrm{m} 2$ among women and $1.1 \mathrm{~kg} / \mathrm{m} 2$ among men, each per decade. BMI have a significant association with chronic diseases (Nasreddine et al.,2012).

In another study done in Malaysia studying the relationship between gender and mean BMI, it was found that males have greater BMI values than females $(22.5 \mathrm{~kg} / \mathrm{m} 2$ vs. $22.3 \mathrm{~kg} / \mathrm{m} 2)$ based on the Second National Health and Morbidities Survey (Khor et al., 1999).

Obesity noted to increase the risk of cardiovascular diseases, mainly heart diseases and stroke (world's number one cause of death that kills 17 million people each year), type 2 diabetes which still is a global epidemic, some cancers such as (endometrial, breast, and colon). Worldwide, a minimum of 2.8 million people die every year as a consequence of being obese or overweight (World Health Organization, 2013).

Obesity is caused by energy imbalance between calories consumed and expended; this can be categorized under behavioral factors such as eating habits and physical inactivity(WHO, 2014). Eating food with high fat content, energy dense food and physical inactivity can cause weight gain and therefore contribute to obesity and overweight condition. Understanding of the relative importance of over consumption along with physical inactivity may help to prevent and reduce the prevalence of obesity and overweight condition among the population.

Other than eating habits and genetic make-up, environmental factors or what can be called 'Obesogenic environment' were shown to be the main reason that leads to the obesity epidemic. Further discussed in this factor, availability of junk food, restaurants, side walk stalls, role of media in food advertisement, and the usage of cars or public transportation over walking or cycling also leads to obesity (Townshend, 2009). Socio-demographic factors such as age, marital status, income and educational level may also cause weight gain and contribute to obesity and overweight status (Center for Disease Control and Prevention, 2001).

According to Ismail et al. (2002), obesity has become a main health concern in Malaysia affecting all age groups and both sexes. Prevalence of overweight and obesity status in Malaysia (14\%) is nearly triple that of China (5.7\%), Japan (5\%), and the Philippines (6.3\%) and double that of Singapore $(7.1 \%)$. This alarming increase in obesity rate in Malaysia cannot be ignored (WHO, NCD Country profile, 2011).

Other than the previously discussed factors, there are few more factors that contributing to increase body weight and obesity. Psychosocial, sleeping pattern, dietary intake and weight control behaviors can be listed as the factors as well. The huge development of food industries in Malaysia also contributing to increased number of overweight and obesity among Malaysians. Food industry developers should start an intervention by producing and contributing more healthy foods (Henderson et al., 2005).

\section{Methods}

The study was conducted in Infrastructure University Kuala Lumpur (IUKL) Malaysia.

\section{Study Design}

This is an analytical cross-sectional study that observes and quantifies factors associated with body-weight status among students in IUKL, it studied interactions between physical activity and environmental factors with body weight status among this population over the defined time. Data were collected from February 2015 to April 2015.

Data Collection An e-mail was sent to the participants a few days before the start of data collection; the email contains information about the researcher, university name, and expectation from the participants. Data collection time was organized with the Student's Affair Department at IUKL. Sampling was randomly selected based on room numbers, using bowl technique. There were three main hostels building, block $\mathrm{F}$ (female), block $\mathrm{G}$ (male), and Lodge block (Male). Lists of empty room numbers were provided which made a total of 147 rooms empty (rejected rooms). A total of 566 room numbers were labelled down in folded 
papers and were placed in a big bowl in such a way that the researcher and assistants couldn't see it. The researcher and assistant kept picking random papers and they went to distribute the questionnaire to the students in the room number that was shown in the paper. After any of the room occupants finished filling the questionnaire, another random number was selected from the bowl. Total number of respondents approached was 311 , empty rooms were 12 , number of students who participated was 281 . Anthropometric measurements such as height, weight, BMI, and waist circumference were collected by the researcher after the participant filled up the questionnaire. Dietary assessment part in the questionnaire was conducted during an interview after filling all previous parts in the questionnaire.

\section{Study Respondents}

The study population consisted of students aged 18 to 65 years old for both males and females, with total number of students 280. Participants who are not around in the campus, younger than 18 years old, participants with physical disability were excluded from participating in this study. The original draft of questionnaire was written in English to be used among international students, and Malay version was created by translating the English to Malay to be used among Malay students. For pre-test, total of 30 questionnaires were distributed among the students in UPM to make sure that the questions were understandable.

\section{Ethical statement}

Before data collection was carried out, ethical approval from the Medical Research Ethics Committee in Universiti Putra Malaysia was obtained to carry out this study. In addition, permission was granted by IUKL to conduct the survey inside IUKL. Before participants filled in the survey, information was given to the participants, once the information was given, a written informed consent was obtained from the participants who agreed to participate in this study.

\section{Data Analysis}

Data were analyzed using Statistical Package For Social Sciences (SPSS) version 21.0. For univariate data, study variables were described using descriptive statistics such as mean, frequencies, standard deviation, and percentages.. Chi square test were performed to test the relationship between categorical independent variables and dependent variable. Meanwhile, t-test was performed to compare mean between continuous variables between obese and non-obese group. Any extreme values were removed to fulfil normal value requirement. A statistical probability level of $\mathrm{p}<0.05$ was considered as significant.

\section{Results}

\section{Study population and socio-demographic characteristics}

A total of $61.2 \%$ of recruited respondents were female $(\mathrm{N}=172)$, while only $38.8 \%$ of respondents were male $(\mathrm{N}=109)$. The majority of respondents in this study were agedin the range of 18-22 years old $(\mathrm{N}=$ $182,64.8 \%$ ), followed by those aged $23-27$ years old (32.4\%). Only 8 respondents in this study were above 28 years old. Respondents aged between 18 to 22 years old were significantly more among female $(p=$ $0.001)$. The mean age of the respondents was $21.72 \pm 2.49$ years, however age was significantly $(t=3.52, p$ $=0.001)$ higher among male $(22.40 \pm 2.89$ vs $21.28 \pm 2.09$ years old $)$. A large percentage of the study respondents were still single at the period of recruitment $(94.3 \%, \mathrm{~N}=265)$. Only $5.7 \%$ of them were married $(\mathrm{N}=16)$. The result also showed that $65.8 \%$ of the respondents were doing their Bachelor degree $(\mathrm{N}=185)$ and this was significantly higher among male $(80.7 \%)$ compared to female $(56.4 \%)$.

Among male, 12.8\% ( $\mathrm{N}=14)$ were completing Foundation studies / A level / Diploma, while 39\% (N=67) of female were doing Foundation / A level / Diploma. The respondents of this study were mainly Malaysians 
$(\chi 2=28.087, \mathrm{p}=0.000)$ and the rest were Non-Malaysians $(55.5 \%$ vs. $44.5 \%)$, especially among the females $(68.0 \%)$.

Most of the students were from the Faculty of Engineering and Technology Infrastructure $(\mathrm{n}=73,26.0 \%)$ and only eight $(2.8 \%)$ of them were from the Faculty of Applied Science and Foundation. The distribution was similar among males. On the other hand, most of the female respondents were from the Faculty of Business and Accounting $(\mathrm{n}=43,25.0 \%)$. The distribution of the study faculty showed significant differences between the genders $(\chi 2=41.61, \mathrm{p}=0.000)$.

\section{Socio-demographic characteristics}

\begin{tabular}{|c|c|c|c|c|c|c|c|}
\hline \multirow[t]{2}{*}{ Variables } & \multirow[t]{2}{*}{ Overall response } & \multicolumn{2}{|c|}{ Gender, N (\%) } & \multirow[b]{2}{*}{$\chi^{2}$} & \multirow{2}{*}{$\begin{array}{c}\text { Fisher's } \\
\text { exact } \\
\text { test }\end{array}$} & \multirow[b]{2}{*}{$t$} & \multirow{2}{*}{$\begin{array}{c}p- \\
\text { value }\end{array}$} \\
\hline & & Male, 109 (38.8) & $\begin{array}{c}\text { Female, } 172 \\
(61.2)\end{array}$ & & & & \\
\hline \multicolumn{8}{|l|}{$\begin{array}{l}\text { Age of respondents } \\
\text { (years) }\end{array}$} \\
\hline $18-22$ & $182(64.8)$ & $60(55.0)$ & $122(70.9)$ & - & 12.28 & - & 0.001 \\
\hline $23-27$ & $91(32.4)$ & $42(38.5)$ & $49(28.5)$ & & & & \\
\hline$\geq 28$ & $8(2.8)$ & $7(6.4)$ & $1(0.6)$ & & & & \\
\hline Mean age \pm SD $($ years $)$ & $21.72 \pm 2.49$ & $22.40 \pm 2.89$ & $21.28 \pm 2.09$ & - & & 3.52 & 0.001 \\
\hline \multicolumn{8}{|l|}{ Marital Status } \\
\hline Single & $265(94.3)$ & $101(92.7)$ & $164(95.3)$ & 0.898 & - & - & 0.430 \\
\hline Married & $16(5.70)$ & $8(7.3)$ & $8(4.7)$ & & & & \\
\hline \multicolumn{8}{|l|}{$\begin{array}{l}\text { Educational } \\
\text { background }\end{array}$} \\
\hline $\begin{array}{l}\text { Foundation/ A level/ } \\
\text { Diploma }\end{array}$ & $81(28.8)$ & $14(12.8)$ & $67(39.0)$ & 22.17 & - & - & 0.000 \\
\hline Bachelor Degree & $185(65.8)$ & $88(80.7)$ & $97(56.4)$ & & & & \\
\hline Master Degree & $15(5.3)$ & $7(6.4)$ & $8(4.7)$ & & & & \\
\hline \multicolumn{8}{|c|}{ Parents' income per month $(\mathrm{RM})^{\mathrm{a}}$} \\
\hline$\leq 2000$ & $16(6.4)$ & $8(8.2)$ & $8(5.3)$ & 3.70 & - & - & 0.448 \\
\hline $2001-4000$ & $51(20.5)$ & $18(18.6)$ & $33(21.7)$ & & & & \\
\hline $4001-6000$ & $42(16.9)$ & $12(12.4)$ & $30(19.7)$ & & & & \\
\hline $6001-8000$ & $35(14.1)$ & $14(14.4)$ & $21(13.8)$ & & & & \\
\hline$\geq 8001$ & $105(42.2)$ & $45(46.5)$ & $60(39.5)$ & & & & \\
\hline $\begin{array}{l}\text { Mean Parents' income } \\
\text { per month } \pm \text { SD }(R M)^{a}\end{array}$ & $\begin{array}{c}\text { RM } 8887.84 \pm \\
\quad 6391.21\end{array}$ & $\begin{array}{l}9547.42 \pm \\
7107.33\end{array}$ & $\begin{array}{c}8466.91 \pm \\
5874.91\end{array}$ & - & & 1.30 & 0.194 \\
\hline \multicolumn{8}{|l|}{ Nationality } \\
\hline Malaysian & $156(55.5)$ & $39(35.8)$ & $117(68.0)$ & 28.087 & & - & 0.000 \\
\hline Non- Malaysian & $125(44.5)$ & $70(64.2)$ & $55(32.0)$ & & & & \\
\hline \multicolumn{8}{|l|}{ Faculty } \\
\hline $\begin{array}{l}\text { Engineering and } \\
\text { Technology Infrastructure }\end{array}$ & $73(26.0)$ & $50(45.9)$ & $23(13.4)$ & - & 41.61 & - & 0.000 \\
\hline $\begin{array}{l}\text { Creative Media and } \\
\text { Innovative }\end{array}$ & $45(16.0)$ & $18(16.5)$ & $27(15.7)$ & & & & \\
\hline Business and Accounting & $57(20.3)$ & $14(12.8)$ & $43(25.0)$ & & & & \\
\hline $\begin{array}{l}\text { Arts, Communication and } \\
\text { Education }\end{array}$ & $51(18.1)$ & $12(11.0)$ & $39(22.7)$ & & & & \\
\hline $\begin{array}{l}\text { Architecture and Built } \\
\text { Environment }\end{array}$ & $47(16.7)$ & $11(10.1)$ & $36(20.9)$ & & & & \\
\hline $\begin{array}{l}\text { Applied Science and } \\
\text { Foundation }\end{array}$ & $8(2.8)$ & $4(3.7)$ & $4(2.3)$ & & & & \\
\hline
\end{tabular}

Note: $\left({ }^{\mathrm{a}}\right)=32$ missing, 


\section{Distribution of physical activity status among study respondents}

The mean total MET per week for study respondents was 3988.50 \pm 6758.45 MET minutes/week. This mean is relatively higher $(\mathrm{t}=3.020, \mathrm{p}=0.003)$ among the male respondents $(5496.37 \pm 7860.26 \mathrm{METs}$ minute/week $)$ compared to female respondents with $3032.93 \pm 5779.55$ METs minutes/week. Generally, all study respondents reported with almost similar percentage of high and low physical activity $(40.2 \%$ and $41.3 \%$ respectively). Across the genders, the majority of males significantly enjoyed high physical activity while females in general preferred low physical activity $\left(\chi^{2}=38.992, p=0.000\right)$. The average time spent for physical activity per day was $108.81 \pm 182.70$ average minute/day. The highest average time was spent on work-related physical activities followed by recreational physical activities and, lastly, travel related physical activities (45.28 $\pm 103.87,34.99 \pm 62.74$ and $28.54 \pm 74.36$ average minutes/day respectively). Male respondents reported significantly higher overall physical activities than females $(t=2.68, \mathrm{p}=$ $0.008)$,especially with level of recreational related physical activities $(t=4.42, \mathrm{p}=0.000)$. Approximately, more than half of the study respondents were not engaged with work-related physical activities (56.9\%) especially females $(61.0 \%$ vs $50.5 \%)$. On the other hand, half of the study male respondents were involved with transports related physical activities (58.7\%). However no significant association was observed.More respondents $(55.2 \%)$ were engaged in recreationally related physical activities in a typical week. Across the genders, males were more significantly engaged in recreational related physical activities $(73.4 \%$ vs $43.6 \%$; $\left.\chi^{2}=23.94, p=0.000\right)$. Similarly, most males were involved in vigorous physical activities $(74.3 \%$ vs $40.7 \%$; $\left.\chi^{2}=30.324, p=0.000\right)$. In a typical day, the study respondents spent $314.67 \pm 22.86$ minutes being sedentary. This study found that females significantly spent moretime for sedentary activities than males $(t=$ - 2.357, $\mathrm{p}=0.019)$.

\section{Distribution of physical activity status among study respondents}

\begin{tabular}{|c|c|c|c|c|c|c|}
\hline \multirow{2}{*}{ Characteristic } & \multirow{2}{*}{$\begin{array}{c}\text { Overall } \\
\text { response } \\
\mathbf{N}(\%)\end{array}$} & \multicolumn{2}{|c|}{ Gender } & \multirow[b]{2}{*}{ t-test } & \multirow[b]{2}{*}{$\chi^{2}$} & \multirow[b]{2}{*}{$p$} \\
\hline & & $\begin{array}{c}\text { Male } \\
\mathbf{N}(\%)\end{array}$ & $\begin{array}{c}\text { Female } \\
\mathbf{N}(\%)\end{array}$ & & & \\
\hline \multicolumn{7}{|c|}{ Total PA (MET-minute/week) } \\
\hline Mean \pm SD & $\begin{array}{c}3988.50 \pm \\
6758.45\end{array}$ & $\begin{array}{c}5496.37 \pm \\
7860.26\end{array}$ & $\begin{array}{c}3032.93 \pm \\
5779.55\end{array}$ & 3.02 & - & 0.003 \\
\hline \multicolumn{7}{|l|}{ Level of total PA } \\
\hline High PA & $113(40.2)$ & $68(62.4)$ & $45(26.2)$ & - & 38.99 & 0.000 \\
\hline Moderate PA & $52(18.5)$ & $8(7.3)$ & $44(25.6)$ & & & \\
\hline Low PA & $116(41.3)$ & $33(30.3)$ & $83(48.3)$ & & & \\
\hline
\end{tabular}

\begin{tabular}{|c|c|c|c|c|c|c|}
\hline \multicolumn{7}{|c|}{ Average time per day (average minute/day) } \\
\hline Total PA & $108.81 \pm$ & $145.01 \pm$ & 85.82 & 2.68 & - & 0.008 \\
\hline$($ Mean \pm SD $)$ & 182.70 & 204.95 & \pm 163.58 & & & \\
\hline $\begin{array}{l}\text { Work related PA } \\
(\text { Mean } \pm \text { SD })\end{array}$ & $\begin{array}{c}45.28 \pm \\
103.87\end{array}$ & $\begin{array}{c}56.28 \pm \\
117.78\end{array}$ & $\begin{array}{c}38.31 \pm \\
93.68\end{array}$ & 1.42 & - & 0.158 \\
\hline $\begin{array}{l}\text { Travel related PA } \\
(\text { Mean } \pm \mathrm{SD})\end{array}$ & $\begin{array}{l}28.54 \pm \\
74.36\end{array}$ & $\begin{array}{c}32.31 \pm \\
77.39\end{array}$ & $\begin{array}{c}26.15 \pm \\
72.49\end{array}$ & 0.68 & - & 0.500 \\
\hline $\begin{array}{l}\text { Recreation related PA } \\
(\text { Mean } \pm \mathrm{SD})\end{array}$ & $\begin{array}{c}34.99 \pm \\
62.74\end{array}$ & $\begin{array}{c}56.49 \pm \\
71.83\end{array}$ & $\begin{array}{c}21.36 \pm \\
52.01\end{array}$ & 4.42 & - & 0.000 \\
\hline
\end{tabular}

Work-related physical activity

\begin{tabular}{|c|c|c|c|c|c|c|}
\hline Did No work & $160(56.9)$ & $55(50.5)$ & $105(61.0)$ & - & 3.05 & 0.081 \\
\hline Did work & $121(43.1)$ & $54(49.5)$ & $67(39.0)$ & & & \\
\hline Did No trans-PA & $130(46.3)$ & 45 (41.3) & $85(49.4)$ & - & 1.78 & 0.183 \\
\hline Did trans PA & $151(53.7)$ & $64(58.7)$ & $87(50.6)$ & & & \\
\hline
\end{tabular}


Recreational-related physical activity

Did No rec-PA $126(44.8)$

Did rec-PA

$155(55.2)$

$$
29(26.6) \quad 97(56.4)
$$

$75(43.6)$

Vigorous PA

Did No vigorous PA

$130(46.3)$

$80(73.4)$

0.000

Did vigorous PA

$151(53.7)$

$28(25.7)$

$102(59.3)$

30.32

0.000

Sedentary activities on average per day (Minutes/day)

Mean \pm SD

$314.67 \pm \quad 275.37 \pm$

222.86

212.40

$339.35 \pm$

226.31

PA-physical activity, SD-standard deviation, MET-metabolic equivalents.

\section{Association between physical activity and body weight status}

Overweight / obese subjects were found highly prevalent among those with low physical activity. However, levels of physical activity never show any trend of association with overweight / obese.The crude analysis found engaging in transport related, recreational related and vigorous physical activity, showed approximately $50 \%$ protection against overweight / obese status(crude OR $=0.58,95 \% \mathrm{CI}=0.35-0.95$, crude $\mathrm{OR}=0.50,95 \% \mathrm{CI}=0.30=0.81$ and crude $\mathrm{OR}=0.42,95 \% \mathrm{CI}=0.26-0.69$ respectively).However, after adjusting for covariate, none of physical activity components showed any association trend with being overweight / obese. In bivariate analysis, sedentary activities showed a very weak association with increased risk of overweight / obese status but not in multivariate analysis (crude OR $=1001,95 \% \mathrm{CI}=1.00-1.002$ ).

\section{Association between physical activity and overweight/obeseamong study respondents}

\begin{tabular}{|c|c|c|c|c|c|c|c|c|c|}
\hline \multirow[b]{2}{*}{ Characteristic } & \multicolumn{2}{|c|}{ BMI } & \multirow[b]{2}{*}{$t$} & \multirow[b]{2}{*}{$\chi^{2}$} & \multirow[b]{2}{*}{$p$} & \multirow[b]{2}{*}{$\begin{array}{c}\text { Crude } \\
\text { OR }\end{array}$} & \multirow[b]{2}{*}{$\begin{array}{c}95 \% \\
\text { CI }\end{array}$} & \multirow[b]{2}{*}{$\mathbf{A O R}^{\mathrm{a}}$} & \multirow[b]{2}{*}{$\begin{array}{c}95 \% \\
\text { CI }\end{array}$} \\
\hline & $\begin{array}{c}\text { Normal } \\
\mathbf{N}(\%) \\
\end{array}$ & $\begin{array}{c}\text { Overweight/ } \\
\text { Obese } \\
\mathrm{N}(\%) \\
\end{array}$ & & & & & & & \\
\hline \multicolumn{10}{|l|}{ Total PA (MET-minute/week) } \\
\hline Mean \pm SD & $\begin{array}{c}4093.9 \pm \\
6198.2\end{array}$ & $\begin{array}{c}3797.6 \pm \\
7699.2\end{array}$ & 0.35 & & 0.726 & 1.00 & $\begin{array}{c}1.00- \\
1.00\end{array}$ & 1.00 & $\begin{array}{c}1.00- \\
1.00\end{array}$ \\
\hline \multicolumn{10}{|l|}{ Level of total PA } \\
\hline High PA & $78(43.1)$ & $35(35.0)$ & & 2.301 & 0.317 & 0.66 & $\begin{array}{c}0.38- \\
1.14\end{array}$ & 0.55 & $\begin{array}{c}0.27- \\
1.11\end{array}$ \\
\hline Moderate PA & $34(18.8)$ & $18(18.0)$ & & & & 0.78 & $\begin{array}{c}0.39- \\
1.54\end{array}$ & 0.46 & $\begin{array}{c}0.20- \\
1.09\end{array}$ \\
\hline Low PA & $69(38.1)$ & $47(47.0)$ & & & & $1^{\mathrm{b}}$ & & $1^{\mathrm{b}}$ & \\
\hline \multicolumn{10}{|l|}{$\begin{array}{l}\text { Average time per day (MET- } \\
\text { minute/day) } \\
\text { Mean } \pm \text { SD }\end{array}$} \\
\hline Total PA & $110.6 \pm 167.1$ & $105.5 \pm 208.9$ & 0.22 & & 0.823 & 1.000 & $\begin{array}{c}0.998- \\
1.001\end{array}$ & 1.00 & $\begin{array}{c}0.998- \\
1.001\end{array}$ \\
\hline Work related PA & $44.2 \pm 93.0$ & $47.2 \pm 121.6$ & $\begin{array}{c}- \\
0.23\end{array}$ & & 0.816 & 1.000 & $\begin{array}{c}0.998- \\
1.003\end{array}$ & 1.00 & $\begin{array}{c}0.997- \\
1.003\end{array}$ \\
\hline Travel related PA & $27.9 \pm 64.6$ & $29.7 \pm 89.7$ & $\begin{array}{c}- \\
0.20\end{array}$ & & 0.844 & 1.000 & $\begin{array}{c}0.997- \\
1.004\end{array}$ & 1.00 & $\begin{array}{c}0.996- \\
1.004\end{array}$ \\
\hline Recreation related PA & $38.5 \pm 66.1$ & $28.6 \pm 55.8$ & 1.27 & & 0.204 & 0.997 & $\begin{array}{c}0.993- \\
1.002\end{array}$ & 0.997 & $\begin{array}{c}0.991- \\
1.002\end{array}$ \\
\hline \multirow{2}{*}{$\begin{array}{l}\text { Work-related physical activity } \\
\text { Did No work } \\
\text { Did work }\end{array}$} & $101(55.8)$ & $59(59.0)$ & & 0.269 & 0.604 & $1^{\mathrm{b}}$ & & $1^{\mathrm{b}}$ & \\
\hline & $80(44.2)$ & $41(41.0)$ & & & & 0.88 & $\begin{array}{c}0.54- \\
1.44\end{array}$ & 0.66 & $\begin{array}{c}0.35- \\
1.22\end{array}$ \\
\hline Transportation-related & $75(41.4)$ & $55(55.0)$ & & 4.767 & 0.029 & $\mathbf{1}^{\mathrm{b}}$ & & $1^{\mathrm{b}}$ & \\
\hline
\end{tabular}


physical activity

Did No trans-PA

Did trans PA

Recreational-related physical activity

Did No rec-PA

Did rec-PA

\section{Vigorous PA}

Did No vigorous PA

Did vigorous PA

Sedentary activities on average per day (Minutes/day)

Mean \pm SD
0.58

0.35

0.95

$0.39-$

1.22

Note: $\left({ }^{\mathrm{a}}\right)=$ Adjusted OR- Estimates of odds ratio from Logistic regression including terms for age, gender, marital status, educational background, parent's income, nationality, faculty, neighbourhood environments for physical activity, emotional eating, sleeping quality, weight control behaviour and energy, $\left({ }^{b}\right)=$ reference group which was a normative group and CI $=$ Confidence interval

\section{Distribution of neighbourhood environments for physical activity among the study respondents}

The overall environmental supports for physical activity among the respondents were at a moderate level $(2.17 \pm 0.72)$, these were similar among both genders, male and female $(t=-0.25 ; p=0.805)$. Almost $27 \%$ of the respondents agreed that their environment did support physical activities. This observation was more obvious among female than male respondents $\left(\chi^{2}=11.513, \mathrm{p}=0.009\right)$. One hundred and eighty four $(65.5 \%)$ respondents strongly agreed that residential density did support PA, with $74.3 \%$ male and $59.9 \%$ of female strongly agreed. However, not many subjects agreed that land use mix supported PA. Transit access was found to somehow support PA $(48.0 \%)$ while pedestrian infrastructure $(84.3 \%)$ strongly supports PA in cases of both male and female respondents, although not significantly different $(89.0 \%$ among male and $81.4 \%$ among female respondents). Bicycling infrastructure was found to be unsupportive of PA (72.2\%). At the same instance, recreational facilities, street connectivity, crime safety, traffic safety, pedestrian safety and aesthetics were found to moderately support physical activities among study respondents (45.9\%. $42.0 \%, 46.6 \%, 44.5 \%, 54.8 \%$ and $51.2 \%$ respectively).

Distribution of neighbourhood environments for physical activity among the study respondents

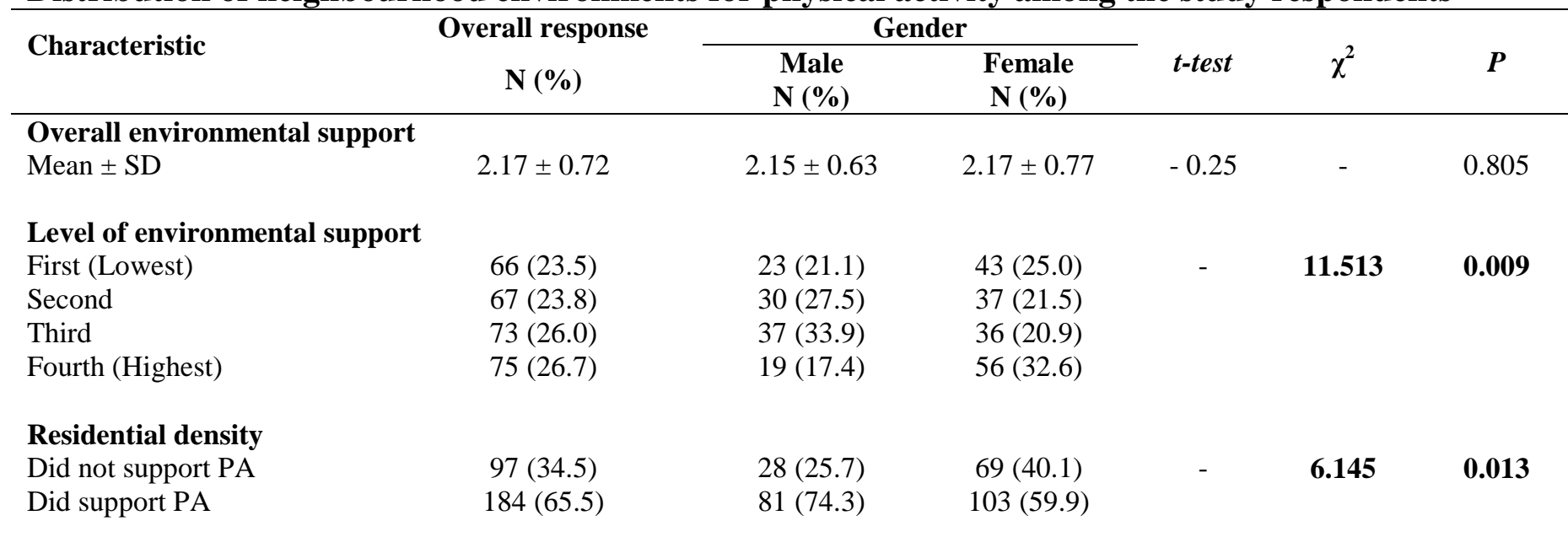


Land use mix

Did not support PA

144 (51.2)

$70(64.2)$

$74(43.0)$

11.998

0.001

Did support PA

$137(48.8)$

$39(35.8)$

$98(57.0)$

Transit Access

Did not support PA

$146(52.0)$

$57(52.3)$

$135(48.0)$

$52(47.7)$

$89(51.7)$

0.008

0.928

Did support PA

$44(15.7)$

$12(11.0)$

$83(48.3)$

Pedestrian infrastructure

Did not support PA

$237(84.3)$

$97(89.0)$

$32(18.6)$

$140(81.4)$

Bicycling infrastructure

Did not support PA

$203(72.2)$

$72(66.1)$

$131(76.2)$

3.399

0.065

Did support PA

$78(27.8)$

37 (33.9)

$41(23.8)$

\section{Recreation facilities}

Did not support PA

$152(54.1)$

$73(67.0)$

129 (45.9)

$36(33.0)$

79 (45.9)

$93(5.1)$

Street connectivity

Did not support PA

$163(58.0)$

64 (58.7)

45 (41.3)

99 (57.6)

73 (42.4)

\section{Crime safety}

Did not support PA

$150(53.4)$

$55(50.5)$

54 (49.5)

95 (55.2)

77 (44.8)

\section{Traffic safety}

Did not support PA

$156(55.5)$

50 (45.9)

$106(61.6)$

66 (38.4)

Pedestrian safety

Did not support PA

127 (45.2)

$59(54.1)$

Did support PA

154 (54.8)

64 (58.7)

$63(36.6)$

109 (63.4)

$\mathbf{1 1 . 8 9 6}$

0.001

$45(41.3)$

$84(48.8)$ $88(51.2)$

Aesthetics

Did not support PA

\section{Association between neighborhood environments for physical activity and body weight status}

Overweight / obese status was highly prevalent among those with low involvement with neighbourhood environment for physical activity (28\%) (the lowest quartile). Although adjusted crude analysis of support to neighbourhood environment for physical activity showed reverse associations with overweight / obese status, no significant trend was observed. 
Table 4.13 Association between neighbourhood environments for physical activity and overweight/obeseamong study respondents

\begin{tabular}{|c|c|c|c|c|c|c|c|c|c|}
\hline \multirow[t]{2}{*}{ Characteristic } & \multicolumn{2}{|c|}{ BMI } & \multirow{2}{*}{ t-test } & \multirow{2}{*}{$\chi^{2}$} & \multirow{2}{*}{$p$} & \multirow{2}{*}{$\begin{array}{c}\text { Crude } \\
\text { OR }\end{array}$} & \multirow{2}{*}{$95 \% \mathrm{CI}$} & \multirow{2}{*}{$\mathbf{A O R}^{\mathrm{a}}$} & \multirow{2}{*}{$95 \% \mathrm{CI}$} \\
\hline & Normal & Overweight & & & & & & & \\
\hline $\begin{array}{l}\text { Overall environmental } \\
\text { support }\end{array}$ & & & & & & & & & \\
\hline Mean \pm SD & $\begin{array}{l}2.2 \pm \\
0.63\end{array}$ & $2.1 \pm 0.85$ & 1.516 & & 0.131 & 0.75 & $\begin{array}{c}0.54- \\
1.06\end{array}$ & 0.77 & $\begin{array}{c}0.51- \\
1.15\end{array}$ \\
\hline
\end{tabular}

Level of environmental support

First (Lowest)

38
$(21.0)$
47

$28(28.0)$

$2.371 \quad 0.499$

$1^{\mathrm{b}}$

$1^{\mathrm{b}}$

Second

47

$20(20.0)$

(26.0)

0.58

$0.28-$

0.49

$0.21-$

$48 \quad 25(25.0)$

Third

(26.5)

0.71

1.18

1.13

Fourth (Highest)

27 (27.0)

$0.36-$

0.58

$0.26-$

0.76

$0.39-$

0.80

1.34

(26.5)

1.51

1.73

Residential density

Did NOT support PA

$63 \quad 34(34.0)$

(34.8)

$66(66.0)$

$118 \quad 66(66.0)$

Did support PA

(65.2)

$0.019 \quad 0.892$

$1^{\mathrm{b}}$

$1^{\mathrm{b}}$

1.04

$0.62-$

1.73

1.04

$0.57-$

1.90

Land use mix

Did NOT support PA

$94 \quad 50(50.0)$

$0.096 \quad 0.756 \quad 1^{\mathrm{b}}$

$1^{\mathrm{b}}$

(51.9)

Did support PA

$87 \quad 50(50.0)$

1.08

$0.66-$

1.16

$0.66-$

(48.1)

1.76

2.06

Transit Access

Did NOT support PA

$93 \quad 53(53.0)$

$0.068 \quad 0.795 \quad 1^{\mathrm{b}}$

$1^{\mathrm{b}}$

(51.4)

Did support PA

$47(47.0)$

(48.6)

$0.94 \quad 0.58-$
1.53

0.95

$0.54-$

1.65

Pedestrian infrastructure

Did NOT support PA

$27 \quad 17(17.0)$

(14.9)

$154 \quad 83(83.0)$

$0.212 \quad 0.646$

$1^{\mathrm{b}}$

$1^{\mathrm{b}}$

Did support PA

(85.1)

0.86

$0.44-$

0.85

$0.38-$

1.66

1.89

Bicycling infrastructure

Did NOT support PA

$132 \quad 71(71.0)$

(72.9)

Did support PA

$49 \quad 29(29.0)$

$0.119 \quad 0.730$

$1^{\mathrm{b}}$

$1^{\mathrm{b}}$

$1.10 \quad 0.64-$

1.10

$0.59-$

1.89

2.05

Recreation facilities

Did NOT support PA

Did support PA

$\begin{array}{cc}96 & 56(56.0) \\ (53.0) & \\ 85 & 44(44.0) \\ (47.0) & \end{array}$

$0.227 \quad 0.633$

$1^{\mathrm{b}}$

$1^{\mathrm{b}}$

$0.54-$

0.93

$0.53-$

1.45

1.63

Street connectivity

Did NOT support PA

$112 \quad 51(51.0)$

$3.129 \quad 0.077$

$1^{\mathrm{b}}$

$1^{\mathrm{b}}$

Did support PA

(61.9)

$69 \quad 49(49.0)$

(38.1)

1.56

$0.95-$

1.51

$0.87-$

2.56

2.62

Crime safety 
Did NOT support PA

Did support PA

Traffic safety

Did NOT support PA

Did support PA

Pedestrian safety

Did NOT support PA

Did support PA

Aesthetics

Did NOT support PA

Did support PA
$60(60.0)$

(49.7)

91

(50.3)

$40(40.0)$

100

(55.2)

81
$(44 / 8)$

$56(56.0)$

$44(44.0)$

$\begin{array}{cc}82 & 45(45.0) \\ (45.3) & \\ 99(54.7) & 55(55.0)\end{array}$

49 (49.0)

0.004

0.951

$51(51.0)$

$2.733 \quad 0.098$

0.66

$0.40-$

0.63

$0.36-$

1.11

$0.015 \quad 0.903$

$1^{\mathrm{b}}$

$1^{\mathrm{b}}$

$0.59-$

0.76

$0.43-$

1.59

1.34

$0.002 \quad 0.961$

$1^{\mathrm{b}}$

$1^{\mathrm{b}}$

1.01

$0.62-$

1.11

$0.63-$

1.65

1.98

Note: $\left({ }^{\mathrm{a}}\right)=$ Adjusted OR- Estimates of odds ratio from Logistic regression including terms for age, gender, marital status, educational background, parent's income, nationality, faculty, physical activity, emotional eating, sleeping quality, weight control behaviour and energy, $\left({ }^{b}\right)=$ reference group which was a normative group and $\mathrm{CI}=$ Confidence interval

\section{Discussion}

Physical activity and its association with body weight status.

Physical inactivity has been acknowledged as a public health concern and has received increasing attention in recent years (Ulaganthan et al., 2012). Findings from this study has contributed important findings on physical activity and its association with obesity.Average time per day for work-related physical activity was found to be higher among obese respondents compared to normal respondents. The study by Kumanan et al, 2013, clearly explains the reason behind this finding, where workload among students to study and to do assignments, which involves more sitting hours, increased the risk of being obese. At the same time, students tend to have their own transport to travel from one place to another place and that reduces their chance to walk or cycle (Muhamod et al., 2013). Due to high BMI or abdominal obesity, student tends to cut down other physical activity that might increase the chance for injuries (Ibrahim et al., 2013). On the other hand, another supportive finding for this is from the study conducted by Mustafa et al (2013) at Hulu Langat, approximately almost half of the respondents reported with low physical activity, meanwhile $48 \%$ of respondents were inactive for more than 6 hours, especially among the obese. Another study entitled Malaysia Aging Male by Ibrahin et al, 2013, findings showed that most of the obese subjects (53.2\%) presented a level of physical activity below 150 minutes 7 days before screening session (insufficiently active).This might due to the individuals with high BMI who reported fear of injuries and having an injury or disease as barriers to physical activity (Ibrahim et al., 2013).

\section{Neighbourhood environments for physical activity and its association with body weight status.}

Over the past decade, a proliferation of interdisciplinary researches have generally supported hypotheses derived from ecological models and identified specific neighborhood environment components and combinations of components that are related to physical activity, mainly for recreation, transportation purposes and obesity (Sallis et al., 2010). In the current study, the neighborhood environment developed for physical activities greatly supports the female gender. Neighborhood environment components such as high 
residential density, high recreation facilities and high traffic safety also support the higher level of physical activities among males. Meanwhile, high land use mix and high pedestrian safety supported higher level of physical activity among female. On the other hand, this neighborhood environment factor shows the same effect among both normal and overweight or obese respondents. However this factor did not support those respondents with abdominal obesity due to their fear of getting injured or even this physical activity might lead to death due to crime. Some studies found that walkable neighborhood is being favorable for obese and overweight people. Somehow, this finding is not supportive in all the studies conducted. But, walkable neighborhood factor is highly supportive for women to involve in physical activity compare to male. Findings from A Carroll-Scott et al, 2013 in his community based study found that a walkable neighborhood improved physical activity of $17 \%$ of respondents especially on vigorous activity.

However, most neighborhoods in Malaysia do not have accessible recreational facilities, such as walking and cycling paths, to encourage physical activity in the community. Physical environment can influence physical activity behaviour level by either promoting or discouraging physical activity through factors such as access to safe recreation, accessibility of recreational facilities, and transit options. Therefore, policy-level interventions and setting of regulations, such as provisions for parks and cycling paths, would help to overcome this problem. Supporting this from findings in this study, one hundred and eighty four (65.5\%) respondents strongly agreed that residential density did support PA, with $74.3 \%$ male and $59.9 \%$ of female strongly agreed, transit access was found to somehow support PA $(48.0 \%)$ while pedestrian infrastructure (84.3\%) strongly supports PA. Bicycling infrastructure was found to be unsupportive of PA (72.2\%). At the same instance, recreation facilities, street connectivity, crime safety, traffic safety, pedestrian safety and aesthetics were found to moderately support physical activities among study respondents (45.9\%. 42.0\%, $46.6 \%, 44.5 \%, 54.8 \%$ and $51.2 \%$ respectively).

\section{Conclusion}

Generally, more than half of the respondents reported with normal BMI and normal waist circumference. The prevalence of abdominal obesity was $32 \%$ which was $29.4 \%$ among male and $33.7 \%$ among females. Work related, recreational related, travel related, vigorous physical activity and being sedentary were significantly higher among female. Meanwhile, only work-related physical activity and being sedentary were found to be predictors for overweight / obese status. However, travel related physical activity only acted as a mild predictor for abdominal obesity among the students in IUKL. Neighborhood environments were highly supportive of physical activity for the female but moderately less supportive for the male subjects

In conclusion, this study provided strong evidence showing significant relationship between physical activity (average time per day on transport-related physical activity, recreational related physical activity, vigorous physical activity and sedentary activity), neighborhood environment physical activity (crime safety) with overweight/obese and abdominal obesity risk. Although adjusted crude analysis of support to neighborhood environment for physical activity showed inverse associations with overweight or obesity, but no significant trend were observed. Findings on the significant relationship between obesity risk and physical activity factors reiterates previously published information.

\section{References}

1. Carroll-Scott, A., Gilstad-Hayden, K., Rosenthal, L., Peters, S. M., McCaslin, C., Joyce, R., \&Ickovics, J. R. (2013). Disentangling neighborhood contextual associations with child body mass index, diet, and physical activity: the role of built, socioeconomic, and social environments. Social Science \& Medicine, 95, 106-114.doi:10.1016/j.socscimed.2013.04.003 
2. Centers for Disease Control and Prevention. (2001). The surgeon general's call to action to prevent and decrease overweight and obesity 2001. Retrieved from http://www.cdc.gov/nccdphp/dnpa/pdf/CalltoAction.pdf

3. Center For Disease Control And Prevention. (2012, March). Adult overweight and obesity. Retrieved from http://www.cdc.gov/obesity/data/adult.html

4. Henderson, V., \& Kelly, B. (2005). Food advertising in the age of obesity: content analysis of food advertising on general market and African American television.37(4), Retrieved from http://www.ncbi.nlm.nih.gov/pubmed/16029689

5. Ibrahim, S., Karim, N. A., Oon, N. L., \&Ngah, W. Z. W. (2013). Perceived physical activity barriers related to body weight status and sociodemographic factors among Malaysian men in Klang Valley. BMC public health, 13(1), 275. doi:10.1186/1471-2458-13-275

6. Ismail, M., Chee, S., Nawawi, H., Yusoff, K., Lim, T., \& James, W. (2002). Obesity in Malaysia. Obesity Reviews, 203-208.

7. Khor, G., Yusof, Azmi., Tee, E Siong., Kandiah, Mirnalini., \& Huang, Mary Soo. (1999). Prevalence of overweight among Malaysian adults from rural communities. Asia Pacific Journal of Clinical Nutrition, 8(4), 272-279.Retrieved December 23, 2013, from http://http://apjen.org/update\%5Cpdf\%5C1999\%5C4\%5C272-279\%5C272.pdf

8. Kumanan J., Yaworski, J., Weber, R. M., \& Ibrahim, N. (2013). What makes students succeed or fail?: The voices of developmental college students. Journal of College Reading and Learning, 30(2), 195-221

9. Mohamud, W. N., Musa, K. I., MdKhir, A. S., Ismail, A. A. S., Ismail, I. S., Kadir, K. A., ... \& Wan Bebakar, W. M. (2011). Prevalence of overweight and obesity among adult Malaysians: an update. Asia Pacific journal of clinical nutrition, 20(1), 35.

10. Mustaffa K.M., Kuczmarski, M. F., Kuczmarski, R. J., \&Najjar, M. (2001). Effects of age on validity of self-reported height, weight, and body mass index: findings from the Third National Health and Nutrition Examination Survey, 1988-1994. Jou of the American Dietetic Association, 101(1), 28-34.

11. Nasreddine, L., Naja, F., Chamieh, M. C., Adra, N., Sibai, A. S., \&Hwalla, N. (2012). Trends in overweight and obesity in lebanon: evidence from two national cross-sectional surveys (1997 and 2009). 798(12), doi: 10.1186/1471-2458-12-798

12. Sallis, J. F., Floyd, M. F., Rodríguez, D. A., \&Saelens, B. E. (2012). Role of built environments in physical activity, obesity, and cardiovascular disease. Circulation, 125(5), 729-737

13. Townshend, T. G. and Lake, A. A. (2009) 'Exploring obesogenic urban form, Theory, policy and practice Health and Place.', Health and Place, 15, (4), pp. 909-916.

14. Ulaganathan, V., Kandiah, M., Zalilah, M. S., Faizal, J. A., Fijeraid, H., Normayah, K., \& Othman, R. (2012). Colorectal cancer and its association with the metabolic syndrome: a Malaysian multi-centric case-control study. Asian Pacific Journal of Cancer Prevention, 13(8), 3873-3877. DOI : 10.7314/APJCP.2012.13.8.387

15. WHO. World Health Organization - NCD Country Profiles, 2011. Geneva: WHO.2011.

16. World Health Organization. (2013, March). Obesity and overweight. Retrieved from http://www.who.int/mediacentre/factsheets/fs311/en/

17. World Health Organisation: Energy Intake (2014)[Online]. Retrieved From : www.who.org.com

18. World Health Organization. (2015, Jannuary). Obesity. Retrieved from http://www.who.int/gho/ncd/risk_factors/obesity_text/en/

19. World Health Organization. (2015, March). Obesity and overweight. Retrieved from http://www.who.int/mediacentre/factsheets/fs311/en 